\title{
Influence de la nature des glucides alimentaires sur l'utilisation de 2 sources protéiques par la truite Arc-en-ciel élevée à $8^{\circ}$ ou $18^{\circ} \mathrm{C}$
}

\author{
P Aguirre, F Medale, SJ Kaushik \\ INRA, station d"hydrobiologie, unité mixte "Nutrition des poissons" INRA-IFREMER, \\ BP 3, 64310 Saint-Pée-sur-Nivelle, France
}

(Reçu le 6 juillet 1994 ; accepté le 24 novembre 1994)

\begin{abstract}
Résumé - L'influence de la nature des glucides alimentaires (amidon de maïs cru ou gélatinisé) sur la valeur biologique d'une farine de poisson (FP) et d'un concentré de protéines solubles de poisson (CPSP) a été testée, par étude du bilan azoté, chez la truite Arc-en-ciel (Oncorhynchus mykiss) élevée à 8 ou $18^{\circ} \mathrm{C}$ pendant $3 \mathrm{sem}$. Les sources protéiques ont été incorporées aux régimes à un taux de $60 \%$, et les sources de glucides à un taux de $30 \%$. L'amidon gélatinisé incorporé dans les régimes a permis d'améliorer la valeur biologique de la farine de poisson (FP) et du CPSP, en réduisant les excrétions azotées des truites alimentées avec ces régimes. Les truites alimentées avec des régimes à base de CPSP ont eu une excrétion azotée plus importante que celles nourries avec les régimes à base de FP, lorsque la source glucidique était de l'amidon cru. La température d'élevage n'a pas modifié la valeur biologique des 2 sources protéiques.
\end{abstract}

nutrition / truite Arc-en-ciel / protéine / amidon / bilan azoté

Summary - Influence of the type of dietary starch on the use of 2 protein sources by rainbow trout reared at 8 or $18^{\circ} \mathrm{C}$. The effect of the type of dietary starch (gelatinized vs native) on the biological value of fish meal (FP) and fish soluble protein concentrate (CPSP) was studied through the nitrogen balance in rainbow trout (Oncorhynchus mykiss) reared for 3 weeks at 2 water temperatures: 8 or $18^{\circ} \mathrm{C}$. The protein sources were included in diets at a level of $60 \%$ and gelatinized or raw starch at a level of $30 \%$. Gelatinized starch improved the biological value of FP and CPSP by reducing the metabolic nitrogen losses. Trout fed the diet with CPSP had a higher nitrogen excretion than those fed the diet with FP. The biological value of both protein sources was unaffected by water temperature.

nutrition / rainbow trout / protein / starch / nitrogen budget

\section{INTRODUCTION}

La valeur biologique (VB), qui reflète le rendement de rétention protéique par rapport à l'azote absorbé, est un critère utilisé pour caractériser la qualité des matières premières protéiques. Dans les aliments piscicoles, les farines de poissons constituent la principale source protéique, leur proportion dans les aliments pour poisson variant de 
25 à $70 \%$ de la matière sèche (Wilson et Halver, 1986). On peut cependant diminuer leur taux d'incorporation dans les aliments en augmentant l'apport d'énergie digestible sous forme de lipides (Watanabe et al, 1979 ; Cho et Kaushik, 1985), ou sous forme de glucides digestibles tels que l'amidon gélatinisé (Kaushik et Oliva-Teles, 1985). Chez les Salmonidés, l'utilisation digestive et métabolique des glucides dépend de leur nature ou de leur complexité (Bergot, 1979a,b ; Palmer et Ryman, 1972 ; Spannhof et Plantikow, 1983). Bergot et Brèque (1983) ont montré que les traitements technologiques (extrusion, gélatinisation) amélioraient la digestibilité des amidons.

De récentes études indiquent que le mode de fabrication des farines de poisson peut induire des différences significatives de croissance chez les Salmonidés (Pike et al, 1990 ; Anderson et al, 1993) vraisemblablement liées à la valeur biologique de ces protéines. C'est pourquoi nous avons comparé les bilans azotés chez des truites nourries avec des aliments contenant comme source protéique soit un concentré de protéines solubles de poisson, soit une farine de poisson couramment utilisée. Nous nous sommes demandé si l'incorporation de glucides digestibles tels que l'amidon gélatinisé pouvait affecter la valeur biologique (VB) de ces sources protéiques. L'influence de la température $\left(8 v s 18^{\circ} \mathrm{C}\right)$, régulateur majeur du métabolisme des poissons (animaux poïkilothermes), a également été étudiée.

\section{MATÉRIEL ET MÉTHODES}

\section{Matières premières protéiques et aliments expérimentaux}

Deux sources protéiques commerciales ont été choisies : l'une était une farine de poisson (FP), l'autre un concentré de protéines solubles de poisson (CPSP). La source protéique FP (pro- téines brutes : $70 \% \mathrm{MS}$ ) était une farine de hareng (Norvège). La source protéique CPSP «Spécial $G$ " (source protéique non dégraissée, protéines brutes : $72 \% \mathrm{MS}$ ), produite par SOPROPECHE (France), est obtenue à partir de poissons entiers ou de déchets de filetage qui, à l'état frais, subissent une hydrolyse enzymatique, une pasteurisation rapide puis un séchage par pulvérisation. La formule des aliments ainsi que leur composition chimique sont décrites dans le tableau I. La fabrication des aliments a été effectuée par pressage à sec (presse Simon-Heese). Une adjonction de $1 \%$ d'oxyde de chrome a été réalisée pour l'étude de la digestibilité.

\section{Matériel animal}

Des truites Arc-en-ciel (Oncorhynchus mykiss) de $80 \mathrm{~g}$ ont été réparties au hasard dans 8 bacs de dimensions 0,5 $\times 0,5 \times 0,2 \mathrm{~m}$. Chaque régime a été testé sur 2 lots de 20 truites. Deux essais ont été réalisés : l'un à $8^{\circ} \mathrm{C}$ et l'autre à $18^{\circ} \mathrm{C}$, dans un circuit d'eau recyclée, filtrée biologiquement et thermorégulée. Les bacs étaient équipés de collecteurs de fèces (Cho et al, 1982). Les animaux ont été nourris manuellement à satiété 2 fois par jour pendant $3 \mathrm{sem}$. La collecte de fèces a été effectuée 2 fois par jour avant chaque repas durant les 2 premières semaines. Les fèces ont été congelées dès la récolte, puis lyophilisées. Les mesures d'excrétion ammoniacale ont été effectuées pendant 3 cycles de $24 \mathrm{~h}$ durant la troisième semaine d'alimentation. Durant ces mesures, la ration a été fixée sur la base de l'ingestion volontaire mesurée au cours des 2 sem précédentes.

\section{Digestibilité}

Les coefficients de digestibilité apparente (CUDa) ont été évalués par la méthode indirecte utilisant l'oxyde de chrome comme marqueur inerte, et calculés selon la formule de Maynard et Loosli (1969).

Dans les aliments comme dans les fèces, l'oxyde de chrome a été dosé par la méthode de Bolin et al (1952). Les protéines brutes ont été analysées par la méthode de Kjeldahl $(\mathrm{N} \times 6,25)$. L'énergie brute a été mesurée à l'aide d'une bombe calorimétrique adiabatique. Les lipides totaux ont été dosés par la méthode de Folch et al (1957) et l'amidon par la méthode de Thivend et al (1972). 
Tableau I. Formule et composition chimique des aliments.

\begin{tabular}{|c|c|c|c|c|}
\hline en $g / k g$ & $C C$ & $C G$ & $F C$ & $F G$ \\
\hline Farine de hareng de Norvège & 0 & 0 & 600 & 600 \\
\hline CPSP a & 600 & 600 & 0 & 0 \\
\hline Amidon de maïs cru & 300 & 0 & 300 & 0 \\
\hline Amidon de maïs gélatinisé & 0 & 300 & 0 & 300 \\
\hline Huile de poisson & 60 & 60 & 60 & 60 \\
\hline Mélange vitaminique ${ }^{b}$ & 10 & 10 & 10 & 10 \\
\hline Mélange minéral $\mathrm{c}$ & 10 & 10 & 10 & 10 \\
\hline Liant (alginate de sodium) & 20 & 20 & 20 & 20 \\
\hline \multicolumn{5}{|l|}{ Composition analytique } \\
\hline Matière sèche (MS) $(\mathrm{g} / \mathrm{kg})$ & 917 & 923 & 885 & 889 \\
\hline Protéines brutes ( $\mathrm{g} / \mathrm{kg} \mathrm{MS}$ ) & 452 & 457 & 413 & 405 \\
\hline Lipides (g/kg MS) & 189 & 189 & 110 & 112 \\
\hline Amidon ( $/ \mathrm{kg}$ MS) & 253 & 255 & 299 & 296 \\
\hline Énergie brute (MJ/kg MS) & 24 & 24 & 21 & 21 \\
\hline Cendres d $(\mathrm{g} / \mathrm{kg} / \mathrm{MS})$ & 23 & 22 & 63 & 76 \\
\hline
\end{tabular}

a Concentré de protéines solubles de poisson (Sopropeche, France). ${ }^{b}$ Composition du mélange vitaminique ( $\mathrm{g} / \mathrm{kg}$ premix) : vitamine A (acétate ; $500000 \mathrm{UI} / \mathrm{g}$ ) : 1 ; vit D3 (DL-cholécalciférol ; $100000 \mathrm{UI} / \mathrm{g}$ ) : 2 ; vit E (DL-alpha tocophérol acétate ; $500 \mathrm{Ul} / \mathrm{kg}$ ) : 5 ; vit $\mathrm{K}$ (sodium ménadione bisulfate) $: 0,3 ;$ thiamine: 0,$75 ;$ riboflavine : 1 ; pyridoxine : 0,75 ; cobalamine : 2,5 ; acide nicotinique : 10 ; acide ascorbique : 25 ; acide folique: 0,25 ; pantothénate de calcium : 2,5 ; chlorure de choline : 100 ; inositol : 50 ; biotine (à $2 \%$ ) : 6 ; cellulose/glucose : $793,95 .{ }^{c}$ Composition du mélange minéral ( $\mathrm{g} / \mathrm{kg}$ premix) : phosphate bicalcique $\mathrm{CaHPO}_{4} \cdot 2 \mathrm{H}_{2} \mathrm{O}: 500$; carbonate de $\mathrm{Ca}\left(\mathrm{CaCO}_{3}\right): 215$; sel marin $(\mathrm{NaCl}): 40$; chlorure de potassium $(\mathrm{KCl}): 90$; hydroxyde de $\mathrm{Mg}\left(\mathrm{Mg}(\mathrm{OH})_{2}\right): 124$; sulfate de fer $\left(\mathrm{FeSO}_{4} .7 \mathrm{H}_{2} \mathrm{O}\right): 20$; sulfate de zinc $\left(\mathrm{ZnSO}_{4} .7 \mathrm{H}_{2} \mathrm{O}\right): 4$; sulfate de cuivre $\left(\mathrm{CuSO}_{4} .5 \mathrm{H}_{2} \mathrm{O}\right): 3$; iodure de potassium $(\mathrm{KI}): 0,04$; sulfate de cobalt $\left(\mathrm{CoSO}_{4}\right): 0,02$; sulfate de manganèse $\left(\mathrm{MnSO}_{4} \cdot \mathrm{H}_{2} \mathrm{O}\right): 3$; sélénite de sodium $\left(\mathrm{Na}_{2} \mathrm{SeO}\right): 0,03$; fluorure de sodium (NaF) : 1. d Obtenues par différence.

\section{Valeur biologique}

Les rendements de rétention azotée par rapport à l'azote absorbé (VB) ont été calculés grâce à la formule suivante (Mitchell, 1924) :

$$
V B=\frac{1-\left(F-F_{O}\right)-\left(U-U_{0}\right)}{1-(F-F o)} \times 100
$$

avec $I=$ azote ingéré ; $F o=$ azote fécal endogène; $F=$ azote fécal ; $U=$ azote urinaire et branchial ; $U_{0}=$ azote urinaire et branchial endogène (mesuré chez des poissons à jeun).

L'azote fécal (F) a été mesuré par analyse du taux d'azote dans les fèces émises par les poissons nourris. La perte d'azote fécal endogène $(F o)$ n'a pas été mesurée dans la présente expérience. Les valeurs rapportées pour la truite Arc- en-ciel (Nose, 1967 ; Roberts, 1976) ou pour la carpe (Ogino et al, 1973) sont de 0,5 à $1,5 \mathrm{mg}$ $\mathrm{N} / \mathrm{g}$ d'aliment sec/jour. En raison de ces faibles valeurs, le fait de ne pas tenir compte de cette perte azotée conduit à une sous-estimation des valeurs de rétention de l'azote absorbé d'environ $2 \%$. L'excrétion ammoniacale, qui représente $85 \%$ des pertes d'azote urinaires et branchiales chez la truite Arc-en-ciel (Kaushik, 1980), a été déterminée, chez les poissons nourris ( 2 lots/régime) ou à jeun (1 lot), en dosant l'azote ammoniacal dans l'eau. Les concentrations en $\mathrm{N}-\mathrm{NH}_{4}$ ont été mesurées dans l'eau d'entrée et de sortie des bacs chaque heure pendant 3 cycles de $24 \mathrm{~h}$ chez les poissons nourris et 1 cycle de $24 \mathrm{~h}$ chez les poissons après $10 \mathrm{j}$ de jeûne. Les dosages ont été réalisés à l'aide d'un auto-analyseur (Technicon) en utilisant la méthode colorimétrique au bleu d'indophénol modifiée de Le Corre et Tréguer (1976). Les débits d'eau de chaque bac ont été mesurés 
toutes les heures. L'excrétion d'ammoniaque par heure et par $\mathrm{kg}$ de poisson a été calculée selon la formule de Kaushik (1980).

\section{Analyse statistique}

Une analyse de variance à 2 facteurs a été réalisée pour tester l'effet du traitement alimentaire, de la température de l'eau et de leur interaction. Lorsqu'un effet du traitement ou de l'interaction était significatif, les moyennes ont été comparées à l'aide du test multiparamétrique de Duncan $(P<0,05)$. Les tests statistiques ont été réalisés à l'aide du logiciel SAS (1987).

\section{RÉSULTATS}

\section{Digestibilités apparentes des nutriments}

Les coefficients de digestibilité apparente des protéines des régimes $C G$ et $C C$ sont plus élevés $(P<0,05)$ que ceux des régimes FG et FC (tableau II). Les coefficients de digestibilité de l'amidon des régimes FG et
CG sont significativement plus élevés $(P<$ $0,05)$ que ceux des régimes $F C$ et $C C$. En revanche, la digestibilité des lipides est semblable $(P>0,05)$ pour tous les régimes quelle que soit la température. Les coefficients de digestibilité apparente de l'énergie des régimes $C G$ et $F G$ sont plus élevés $(P<$ $0,05)$ que ceux des régimes $C C$ et $F C$ respectivement.

Les CUDa de la matière sèche, de l'énergie et de l'amidon sont plus élevés à $18^{\circ} \mathrm{C}$ qu'à $8^{\circ} \mathrm{C}(P<0,05)$. En revanche, la température de l'eau n'influence pas significativement les CUDa des protéines ou des lipides. En conséquence le rapport protéines digestibles/énergie digestible diffère pour un même régime en fonction de la température d'élevage des poissons (tableau III).

\section{Consommation et excrétion azotée des poissons}

Les consommations d'azote ainsi que les pertes azotées fécales et les pertes méta-

Tableau II. Coefficient d'utilisation digestive apparente des nutriments (CUDa) à 8 et $18^{\circ} \mathrm{C}$.

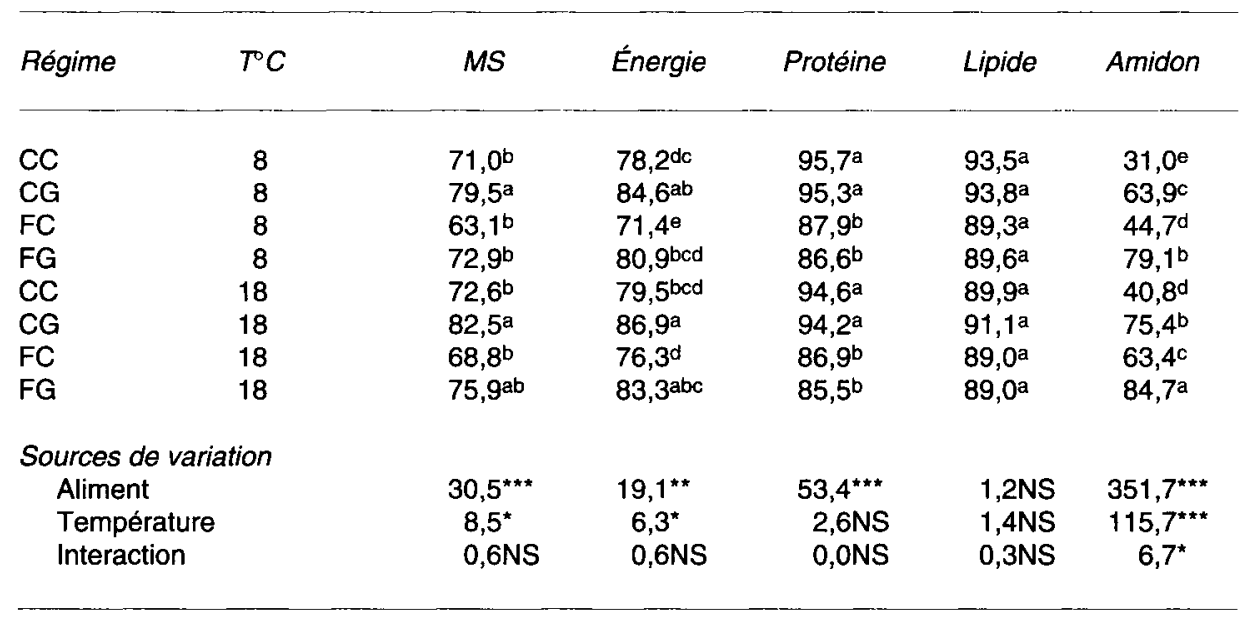

Dans chaque colonne, les moyennes avec des lettres différentes sont significativement différentes $(P<0,05)$. ${ }^{\star} P<0,05 ;{ }^{* *} P<0,01 ;{ }^{\star \star \star} P<0,001 ;$ NS : $P>0,05$. 
boliques (urinaires et branchiales) d'azote ammoniacal sont présentées dans le tableau IV. Les pertes azotées fécales sont significativement plus élevées chez les truites alimentées avec les régimes à base de FP que chez les truites nourries avec les régimes à base de CPSP. La source glucidique n'influence pas significativement les excrétions azotées fécales des truites, quelle que soit la source protéique. La température de l'eau augmente significativement les quantités d'azote fécal excrétées par les truites.

L'excrétion ammoniacale branchiale et urinaire des truites nourries avec les régimes à base de CPSP est significativement supérieure à celle des truites nourries avec les régimes à base de FP aux 2 températures d'élevage et pour chaque source de glucide. Cependant l'excrétion ammoniacale exprimée en fonction de l'azote absorbé est plus élevée avec le CPSP seulement lorsque cette source protéique est associée à l'amidon cru dans le régime.

À $18^{\circ} \mathrm{C}$, les excrétions branchiales et urinaires sont plus faibles $(P<0,05)$ chez les truites nourries avec les régimes contenant l'amidon gélatinisé que chez celles qui sont nourries avec les régimes correspondants contenant de l'amidon cru. En revanche à $8^{\circ} \mathrm{C}$, ceci n'est observé qu'avec les régimes à base de CPSP. L'excrétion azotée journalière des truites (exprimée par unité de poids vif) est significativement plus élevée à $18^{\circ} \mathrm{C}$ qu'à $8^{\circ} \mathrm{C}$.

\section{Valeur biologique (VB) (tableau V)}

La présence d'amidon gélatinisé dans les régimes ( $F G$ et $C G$ ) induit une augmentation de la VB des protéines quelle que soit la source protéique, aux 2 températures. $L a$ VB de la farine de poisson n'est pas différente $(P>0,05)$ de celle du CPSP lorsque la source glucidique est de l'amidon gélatinisé. Associée à de l'amidon cru, la farine de poisson a une VB supérieure $(P<0,05)$ à celle du CPSP associé à de l'amidon cru.

La VB de chacun des régimes expérimentaux n'est pas significativement influencée par la température.

\section{DISCUSSION}

La digestibilité des protéines du CPSP est supérieure à celle des protéines de la farine de poisson. Les propriétés physico-chimiques du CPSP, différentes de celles de la

Tableau III. Protéines digestibles et énergie digestible des régimes en fonction de la température d'élevage des truites.

\begin{tabular}{lcccr} 
en $g / k g$ & $C C$ & $C G$ & $F C$ & $F G$ \\
\hline Température : $8^{\circ} \mathrm{C}$ & & & & \\
$\quad$ Protéines digestibles (\% MS) & 43,3 & 43,5 & 36,3 & 35,1 \\
Énergie digestible (kJ/g MS) & 18,8 & 20,3 & 15,0 & 17,0 \\
DP/DE (mg/kJ) & 23,1 & 21,5 & 24,2 & 20,6 \\
& & & & \\
Température : $18^{\circ} \mathrm{C}$ & 42,8 & 43,0 & 35,9 & 34,6 \\
Protéines digestibles (\% MS) & 19,1 & 20,9 & 16,0 & 17,5 \\
Énergie digestible (kJ/g MS) & 22,4 & 20,6 & 22,4 & 19,8 \\
DP/DE (mg/kJ) & & & &
\end{tabular}


Tableau IV. Consommation et excrétion azotée des poissons.

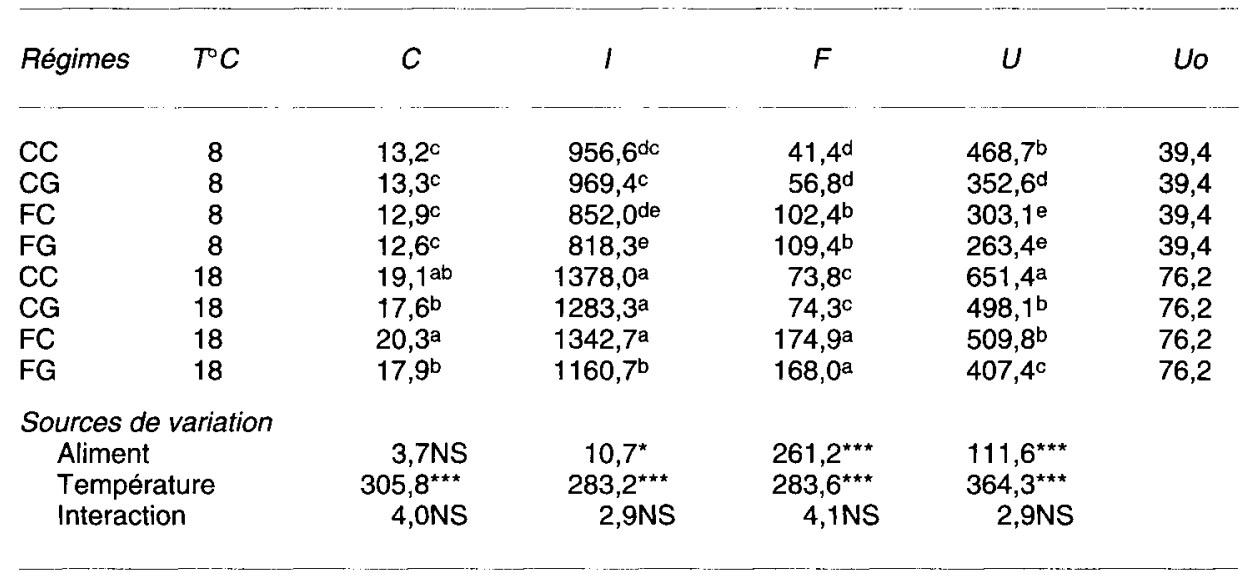

$\mathrm{T}^{\circ} \mathrm{C}$ : température ; $\mathrm{C}$ : consommation d'aliment $\mathrm{g} / \mathrm{kg} \mathrm{PV} / \mathrm{j} ; \mathrm{U}$ : azote urinaire et branchial $(\mathrm{mg} \mathrm{N} / \mathrm{kg} \mathrm{PV} / \mathrm{j}) ; \mathrm{Uo}$ : azote urinaire et branchial endogène $(\mathrm{mg} \mathrm{N} / \mathrm{kg} P V / j) ; 1$ : azote ingéré (mg N/kg PV/j) ; F : azote fécal $(\mathrm{mg} \mathrm{N} / \mathrm{kg}$ $\mathrm{PV} / \mathrm{j})$. Dans chaque colonne, les moyennes avec des lettres différentes sont significativement différentes $(P<$ $0,05) .{ }^{*} P<0,05 ;{ }^{* \star *} P<0,001 ;$ NS : $P>0,05$.

FP, peuvent expliquer une telle différence. En effet, le CPSP a une granulométrie plus faible que la farine de poisson, une plus grande solubilité $(80 \%)$, une teneur en cendres 2 fois moins importante (tableau

Tableau V. Valeur biologique des protéines des régimes expérimentaux.

\section{Régimes Température Valeur biologique}

$\begin{array}{lrl}\mathrm{CC} & 8 & 53,0^{\mathrm{d}} \\ \mathrm{CG} & 8 & 66,0^{\mathrm{abc}} \\ \mathrm{FC} & 8 & 64,8^{\mathrm{bc}} \\ \mathrm{FG} & 8 & 68,4^{\mathrm{a}} \\ \mathrm{CC} & 18 & 55,8^{\mathrm{d}} \\ \mathrm{CG} & 18 & 65,1^{\mathrm{bc}} \\ \mathrm{FC} & 18 & 62,8^{\mathrm{c}} \\ \mathrm{FG} & 18 & 66,6^{\mathrm{ab}}\end{array}$

Sources de variation

$\begin{array}{lr}\text { Aliment } & 78,7^{* * *} \\ \text { Température } & 0,4 \mathrm{NS} \\ \text { Interaction } & 2,9 \mathrm{NS}\end{array}$

Dans chaque colonne, les moyennes avec des lettres différentes sont significativement différentes $(P<0,05)$. ${ }_{\star \star \star \star}: P<0,001 ;$ NS : $P>0,05$.
II) et contient des peptides courts et des acides aminés libres (données du fabricant). Les valeurs de CUDa des protéines pour le CPSP et pour la farine de poisson sont en accord avec celles rapportées par Kaushik et Médale (1994) et indiquent qu'il s'agit de matières premières de bonne qualité.

Le coefficient de digestibilité apparente de l'amidon gélatinisé est deux fois plus élevé que celui de l'amidon cru confirmant l'amélioration obtenue par les traitements technologiques (Bergot et Brèque, 1983). Ceci explique que les coefficients de digestibilité apparente de la matière sèche et de l'énergie soient significativement plus élevés dans les régimes à base d'amidon gélatinisé. En conséquence, les régimes contenant de l'amidon gélatinisé apportent davantage d'énergie digestible que les régimes contenant de l'amidon cru (tableau III). La nature de l'amidon (cru ou gélatinisé) incorporé à l'aliment n'a pas d'influence sur la digestibilité des lipides et des protéines. Ainsi, les pertes fécales azotées ne sont influencées que par la nature de la source protéique. 
L'augmentation de la température d'élevage améliore la digestibilité des glucides. Ce résultat suggère que l'activité amylasique est sans doute influencée par la température de l'eau chez la truite, comme cela a été observé chez le gardon, Rutilus rutilus (Hofer, 1979). En revanche, la digestibilité des autres nutriments n'est pas significativement modifiée par la température, en accord avec les résultats observés par Cho et al (1982). De ce fait, le rapport protéines digestibles/énergie digestible des régimes varie avec la température (tableau III).

À $18^{\circ} \mathrm{C}$, les truites ont consommé en moyenne $44 \%$ d'aliment de plus qu'à $8^{\circ} \mathrm{C}$. L'élévation de température augmente l'activité des poissons ainsi que le métabolisme de base ; pour cela les poissons réajustent leur consommation alimentaire en fonction de leurs besoins énergétiques (Cho, 1990). Les quantités d'azote rejetées dans les fèces et par l'urine et les branchies augmentent proportionnellement.

Les pertes d'azote ammoniacal représentent 30 à $50 \%$ de l'azote ingéré. Le remplacement de l'amidon cru par l'amidon gélatinisé dans les régimes à base de CPSP comme de FP permet de réduire les rejets azotés d'origine métabolique des poissons comme cela avait déjà été montré par Kaushik et Oliva-Teles (1985). Cette diminution du catabolisme azoté démontre l'effet bénéfique de la diminution du rapport DP/DE du régime, résultant de l'augmentation de l'apport d'énergie digestible non-protéique, sur la rétention azotée.

La quantité d'azote ammoniacal excrétée par les truites recevant les régimes à base de CPSP est toujours supérieure (de 18 à $35 \%$ ) à celle des truites nourries avec les régimes à base de FP, quelle que soit la source glucidique du régime. Cette différence ne résulte pas seulement de l'augmentation de la quantité d'azote digestible apporté par le CPSP, dont les protéines sont hautement digestibles (CUDa > 94\%). En effet, l'excrétion ammoniacale, expri- mée en pourcentage de l'azote absorbé, est plus élevée chez les truites recevant le régime à base de CPSP $(50,6 \%)$ que chez celles nourries avec le régime à base de farine de poisson (42\%), lorsque ces sources protéiques sont associées à de l'amidon cru. En conséquence, la valeur biologique du régime combinant CPSP et amidon cru est la plus faible quelle que soit la température. En revanche la valeur biologique des 2 sources protéiques n'est pas différente lorsque le régime contient de l'amidon gélatinisé $(39,1$ et $39,9 \%$ respectivement pour FP et CPSP). Le CPSP comporte davantage de petits peptides et d'acides aminés libres que la farine de poisson. L'absorption intestinale des acides aminés pourrait donc être plus rapide et l'apport des acides aminés dans le sang plus massif chez les truites recevant le régime à base de CPSP que chez celles nourries avec le régime à base de farine de poisson. Ce phénomène pourrait entraîner un catabolisme du surplus d'acides aminés, et donc une excrétion d'azote plus importante, chez les truites nourries avec les régimes à base de CPSP lorsque le rapport DP/DE est supérieur à $22 \mathrm{mg} / \mathrm{kJ}$. Ces observations suggèrent que la substitution totale de la farine de poisson par du CPSP dans les aliments piscicoles peut induire une diminution de la rétention azotée. D'ailleurs, Langar (1992) a observé que la rétention azotée chez le bar diminuait lorsque le taux d'incorporation du CPSP dans le régime augmentait de 18 à $30 \%$ (CUP de 22 vs 27\%).

\section{CONCLUSION}

La présente étude démontre que la valeur biologique des sources protéiques dépend du taux énergétique du régime mais aussi de l'équilibre entre protéines digestibles et énergie digestible (rapport DP/DE). L'amidon gélatinisé, en apportant de l'énergie diges- 
tible, permet une épargne d'azote significative ainsi que l'exprime la VB des 2 sources protéiques. Dans ce cas, les 2 sources protéiques testées ne peuvent pas être différenciées en termes de rendement de rétention d'azote absorbé. En revanche, lorsque le rapport protéines digestibles/énergie digestible du régime est supérieur à $22 \mathrm{mg} / \mathrm{kJ}$ (régimes contenant l'amidon cru comme source glucidique), le catabolisme des protéines du CPSP est plus intense et le rendement de rétention de l'azote absorbé plus faible. Enfin, la température d'élevage ne modifie pas significativement la valeur biologique de ces sources protéiques alimentaires.

\section{RÉFÉRENCES}

Anderson JS, Lall SP, Anderson DM, McNiven MA (1993) Evaluation of protein quality in fish meals by chemical and biological assays. Aquaculture 115, 305-325

Bergot $F$ (1979a) Carbohydrates in rainbow trout diets: effets of the level and source of carbohydrates and the number of meals on growth and body composition. Aquaculture 18, 157-167

Bergot $F(1979 b)$ Effects of dietary carbohydrates and their mode of distribution on glycaemia in rainbow trout (S gairdneri R). Comp Biochem Physiol 64A, 543-547

Bergot $F$, Brèque $J(1983$ ) Digestibility of starch by rainbow trout: effect of the physical state of starch and of the intake level. Aquaculture 34, 203-212

Bolin DW, King RP, Klosterman WW (1952) A simplified method for the determination of chromic oxide $\left(\mathrm{Cr}_{2} \mathrm{O}_{3}\right)$ when used as an inert substance. Science 16, 634-635

Cho CY (1990) Fish nutrition, feeds and feeding: with special emphasis on salmonid aquaculture. Feed Rev Int 6, 333-357

Cho CY, Kaushik SJ (1985) Effects of protein intake on metabolizable and net energy values of fish diets. Nutrition and Feeding in Fish (CB Cowey, AM Mackie, JG Bell, eds), Academic Press, London, 95-117

Cho CY, Slinger SJ, Bayley HS (1982) Bioenergetics of salmonid fishes: energy intake, expenditure and productivity. Comp Biochem Physiol 73B, 25-41

Folch J, Lees M, Sloane Stanley GH (1957) A simple method for the isolation and purification of total lipids from animal tissues. J Biol Chem 226, 497-509

Hofer R (1979) The adaptation of digestive enzymes to temperature, season and diet in roach, Rutilus ruti-
IUS L and rudd, Scardinus erythrophtalmus L. 1. Amylase. J Fish Biol 14, 565-572

Kaushik SJ (1980) Influence of nutritional status on the daily pattern of nitrogen excretion in the carp (Cyprinus carpio $\mathrm{L}$ ) and the rainbow trout (Salmo gairdneri L). Reprod Nutr Dev 20, 6, 1751-1765

Kaushik SJ, Oliva-Teles A (1985) Effect of digestible energy on nitrogen and energy balance in rainbow trout. Aquaculture 50, 89-101

Kaushik SJ, Médale F (1994) Energy requirements, utilization and dietary supply to salmonids. Aquaculture 124, 81-97

Langar $H$ (1992) Effets physiologiques et métaboliques de la qualité nutritionnelle des protéines chez le jeune alevin de bar (Dicentrarchus labrax). Thèse de doctorat, univ de Bretagne Occidentale, $136 p p$

Le Corre $P$, Tréguer $P$ (1976) Contribution à l'étude de la matière organique dissoute et des sels nutritifs dans l'eau de mer. Caractéristiques chimiques du golfe de Gascogne et des upwellings côtiers de I'Afrique du Nord-Ouest. Thèse d'état, univ de Bretagne Occidentale, Brest, $490 \mathrm{pp}$

Maynard LA, Loosli JK (1969) Animal Nutrition (6e ed). McGraw-Hill, New York, États-Unis

Mitchell HH (1924) The nutritive value of proteins. Physiol Rev 4, 424-478

Nose T (1967) On the metabolic fecal nitrogen in young rainbow trout. Bull Freshwat Fish Res Lab 17, 97-105

Ogino C, Kakino I, Chen MS (1973) Protein nutrition in fish. Il. Determination of metabolic fecal nitrogen and endogenous nitrogenous excretion of carp. Bull Jap Soc Sci Fish 35, 519-523

Palmer TN, Ryman BE (1972) Studies on oral glucose intolerance in fish. J Fish Biol 4, 311-319

Pike IH, Andorsdottir G, Mundheim H (1990) The role of fish meal in diet for salmonids. International Association of fish meal manufacturers, Hertfordshire, UK $n^{\circ} 24,35 p p$

Roberts JK (1976) The metabolism and growth of rainbow trout in fresh and saline waters. PhD thesis, univ Aston, Royaume-Uni, $327 \mathrm{p}$

SAS (1987) Statistical guide for personal computers. SAS Inst Inc, NC, États-Unis, $1028 \mathrm{p}$

Spannhof L, Plantikow H (1983) Studies on carbohydrate digestion in rainbow trout. Aquaculture 30, 95-108

Thivend P, Mercier C, Guilbot A (1972) Determination of starch with glucoamylase. In : Methods in Carbohydrates Chemistry (RI Whistler, JN Bemiller, eds), Academic Press, New York, vol VI, 100-105

Watanabe T, Takeuchi T, Ogino C (1979) Studies on the sparing effects of lipids on dietary protein in rainbow trout ( $S$ gairdneri). In : Finish Nutrition and Fishfeed Technology (K Tiews, JE Halver, eds), vol I, Heenemann, Berlin, 113-125

Wilson RP, Halver JE (1986) Protein and amino acid requirements of fishes. Ann Rev Nutr 6, 225-244 